International Journal of Engineering \& Technology, $7(2.21)(2018) 144-150$
International Journal of Engineering \& Technology
SPC
Website: www.sciencepubco.com/index.php/IJET
Research paper

\title{
Static, Transient and Free Vibirational analysis of thermo magneto electric elastic solid cylinder
}

\author{
S. Karthikeyan ${ }^{1 *}$, T.K. Parvatha Varthini ${ }^{2}$ \\ ${ }^{1}$ Department of Mathematics, Government Arts College (Autonomous), Salem \\ ${ }^{2}$ Department of Mathematics, Sona College of Technology (Autonomous), Salem \\ *Corresponding author E-mail: rishabkarthik1@gmail.com
}

\begin{abstract}
In this paper the static, transient and free vibration analysis of a thermo- magneto-electric-elastic solid cylinder is analyzed stochastically by using hybrid numerical method (combined finite element and Newmark finite difference method). An infinite solid cylinder made up of $6 \mathrm{~mm}$ class considered. The constitutive equations containing the mechanical, magnetic, electrical and thermal fields and investigated by free and forced Vibirational boundary conditions. The transient finite element equations are obtained by assumed shape functions. After assembling the Mass, Stiffness and Damping and matrices, the global dynamic equations are in the form of time field. The resulting equations are solved by using the finite difference technique with suitable time instants. By using material constants values the displacement, velocity and acceleration of vibrations are obtained with various time values and the non dimensional frequencies are also obtained by different values of non dimensional wave number. Numerical work is carried out by the electric and magnetic materials Cdse and $\mathrm{CoFe} 2 \mathrm{o} 4$. The outcomes are tabulated and represented graphically.
\end{abstract}

Keywords: Static/Transient/Free analysis, thermo-magneto-electric-elastic, Solid cylinder, hybrid numerical method, Cdse and CoFe2o4, displacement, Velocity, acceleration, non dimensional frequencies, dispersion curves.

AMS subject classification: $74 K 20,74 G 15,74 S 05$.

\section{Introduction}

The dynamic behaviour and wave propagation in structures have been used to safety evaluation of stress field in engineering structures.

High frequency vibration of Piezoelectric crystal plates has been studied by [1]. Wave propagation in Piezo composite plate have analysed by [2].The vibration of pyro electric sandwich plate has studied by [3].Free vibrations of pyro electric layer hexagonal $(6 \mathrm{~mm})$ class has analysed by [4].Vibration of pyro electric plates are discussed by [5]. In [6] dealt with free vibrations of Piezo electric layer of Hexagonal $(6 \mathrm{~mm})$ class. The free vibration response of two dimensional magneto-electro-elastic laminated plates has been studied by [7]. The Finite Element Modelling of a Layered, Multiphase Magneto electro elastic cylinder subjected to an ax symmetric temperature Distribution has been studied by [8].Galerkin finite element derivation for vibration of a thermo piezoelectric structure have been analysed by [9]. Transient analysis of thermo elastic waves in thick hollow cylinders using a

$$
\begin{array}{ll}
T_{k j}=C_{k j r s} S_{r s}-e_{r k j} E_{r}-d_{k i} H_{k}-\beta_{k j} T, & S_{k j}=\frac{1}{2}\left(u_{k, j}+u_{j, k}\right), \\
D_{k}=e_{k l r} S_{l r}+\varepsilon_{k l} E_{l}-m_{l k} H_{k}+P_{k} T, & E_{k}=-\varphi_{, k}, \\
B_{k}=d_{j i} S_{r s}+m_{l k} E_{k}+\mu_{l k} H_{k}+\tau_{l} T & \\
\eta=\beta_{k j} S_{k j}+P_{k} E_{k}+\tau_{l} H_{k}+a T, & a=\rho C_{v} T_{0}^{-1}, \quad q_{k}=-k_{k j} T_{j}
\end{array}
$$

stochastic hybrid numerical method, considering Gaussian mechanical properties is studied by [10].On calculating dispersion curves of waves in a functionally graded elastic plate have been analyzed by [11]. Meshless local petrov-Galerkin method for coupled thermo elasticity analysis of a functionally graded thick hollow cylinder has discussed with [12].Thermoelastic wave propagation in functionally graded materials using meshless local petrov-Galerkin(MLPG) method has studied by [13]. Exact solutions for magneto electro elastic laminates in cylindrical bending is studied by [14].Exact solution for simply supported and multi latered Magneto-Electro-Elastic plates is analyzed by [14].In [15],[16] had given a MATLAB algorithm for finite element numerical implementation.

\section{Governing equation}

The equations of linear thermo piezoelectricity were proposed by Mindlin [1].The governing equations are,

Where $T_{k j}$ and $S_{k j}$ are the stress and strain tensors, the remaining terms $u_{k}, D_{k}, E_{k}$ and $H_{k}$ are mechanical displacement, the electric displacement, the electric and magnetic fields respectively. $B_{k}, d_{j i}, m_{l k}, \mu_{l k}$ and $\tau_{l}$ are magnetic

induction, piezo magnetic, magneto electric, magnetic permeability and pyromagnetic coefficients respectively. $T$ is the temperature change from a reference temperature $T_{0}, q_{i, j}$ the heat flux, $\phi$ the electric potential, $\eta$ the entropy, $\rho$ the mass 
density and $C_{k j r s}, e_{r k j}, \beta_{k j}, \varepsilon_{k j}, p_{k}$ are the elastic, piezoelectric, stress coefficient, dielectric, pyroelectric material constants respectively and $C_{v}$ the specific heat. The usual notation is displayed, and the comma followed by a lower case denotes partial differentiation of that coefficient with respect to independent variable.

The assumed solution for an infinite solid cylinder along with $(l, m, 0)$ direction with cylindrical polar coordinates $(r, \theta, z, t)$ are as, by [7]

$$
\begin{aligned}
& u_{1}(r, \theta, z, t)=U(r) \exp i(k l z+k m \theta-\omega t) \\
& u_{2}(r, \theta, z, t)=V(r) \exp i(k l z+k m \theta-\omega t) \\
& u_{3}(r, \theta, z, t)=\left(\frac{i}{h}\right) W(r) \exp i(k l z+k m \theta-\omega t) \\
& \Phi(r, \theta, z, t)=i\left(\frac{C_{44}}{e_{33}}\right)\left(\frac{\Phi(r)}{h}\right) \exp i(k l z+k m \theta-\omega t) \\
& \psi(r, \theta, z, t)=i\left(\frac{C_{44}}{d_{33}}\right)\left(\frac{\Psi(r)}{h}\right) \exp i(k l z+k m \theta-\omega t) \\
& \Theta(r, \theta, z, t)=\left(\frac{C_{44}}{\beta_{33}}\right)\left(\frac{\Theta(r)}{h}\right) \exp i(k l z+k m \theta-\omega t)
\end{aligned}
$$

Where $k$ is the wave number and $\omega$ is frequency. $l=\cos \theta, m=\sin \theta, l^{2}+m^{2}=1$ and $i=\sqrt{-1}$. Introduce the non dimensional wave number $\varepsilon=k h$ ( $h$ is the thickness of the cylinder).

\section{Boundary condition}

In traction free boundary conditions, the body force and the densities are absence. By using these, the governing equations can bewritten as,

$$
\begin{gathered}
T_{k j, j}=\rho \ddot{u}_{k} \\
D_{k, k}=\mathrm{O}, \\
B_{k, k}=\mathrm{O} \\
q_{k, k}+T_{\mathrm{o}} \dot{\eta}=\mathrm{O}
\end{gathered}
$$

The equation (1) can be rewritten as,

$$
\begin{aligned}
& u_{1}(r, \theta, z, t)=z^{j-1} \exp i(k l z+k m \theta-p t)\{U\} \\
& u_{2}(r, \theta, z, t)=z^{j-1} \exp i(k l z+k m \theta-p t)\{V\} \\
& u_{3}(r, \theta, z, t)=\left(\frac{i}{h}\right) z^{j-1} \exp i(k l z+k m \theta-p t)\{W\} \\
& \Phi(r, \theta, z, t)=i\left(\frac{C_{44}}{e_{33}}\right)\left(\frac{1}{h}\right) z^{j-1} \exp i(k l z+k m \theta-\omega t)\{\Phi\} \\
& \psi(r, \theta, z, t)=\left(\frac{C_{44}}{e_{33}}\right)\left(\frac{i}{h}\right) z^{j-1} \exp i(k l z+k m \theta-p t)\{\Psi\} \\
& \Theta(r, \theta, z, t)=\left(\frac{C_{44}}{\beta_{33}}\right)\left(\frac{1}{h}\right) z^{j-1} \exp i(k l z+k m \theta-p t)\{\Theta\}
\end{aligned}
$$

$$
\left.\begin{array}{l} 
\\
H_{k}-\beta_{k j} T=\rho \ddot{u}_{k}, \\
m_{l k} H_{k}+P_{k} T=0, \\
+\mu_{l k} H_{k}+\tau_{l} T=0 \\
\tau_{l} H_{k}+a T-\frac{k_{k l}}{T_{0}} T_{, k l}=0
\end{array}\right]
$$

\section{Finite element technique}

The assumed shape functions are required to construct the finite element formulation, that the corresponding mechanical, magnetic, electrical and thermal fields are as follows,

$$
\left.u_{i}=\left[N_{u}^{e}\right]\left\{u^{e}\right\}, \Phi=\left[N_{\phi}^{e}\right]\left\{\phi^{e}\right\}, \psi=\left[N_{\psi}^{e}\right]\left\{\psi^{e}\right\}, \quad \Theta=\mid N_{\Theta}^{e}\right]\left\{\Theta^{e}\right\}
$$

Where $\left\{u^{e}\right\},\left\{\psi^{e}\right\},\left\{\phi^{e}\right\}$ and $\left\{\Theta^{e}\right\}$ are unknown nodal points and $\left.\left\lfloor N_{u}^{e}\right\rfloor, \mid N_{\phi}^{e}\right\rfloor,\left\lfloor N_{\psi}^{e}\right\rfloor$ and $\left\lfloor N_{\Theta}^{e}\right\rfloor$ are corresponding shape functions and $e$ is the element level degrees of freedom.

wave propagation of an infinite solid cylinder, along the thickness direction, Power series are most commonly used for shape functions.

The solution of an infinite solid cylinder can be assumed as,
Substitute the free boundary condition, shape function, equation (5) in (4) and to make integrate over the suitable corresponding volume. Obtain a completely coupled system of the following
Global Stiffness $[K]$, Damping $[C]$ and Mass $[M]$ matrices are, 


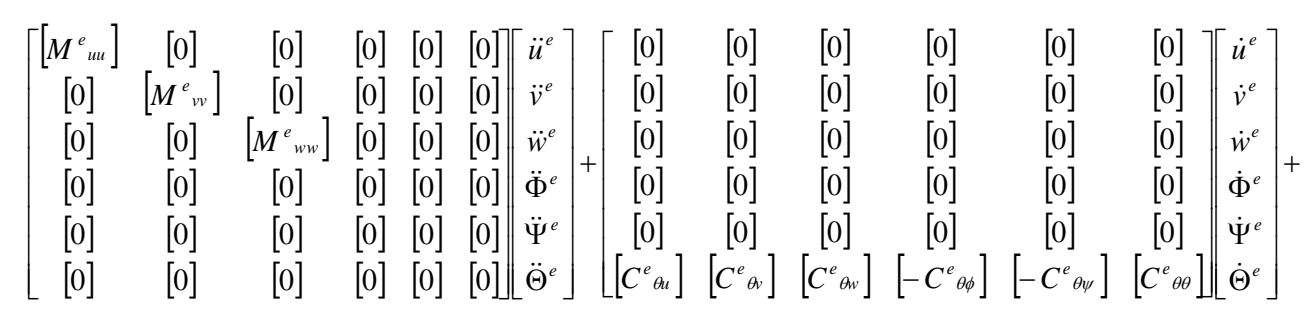

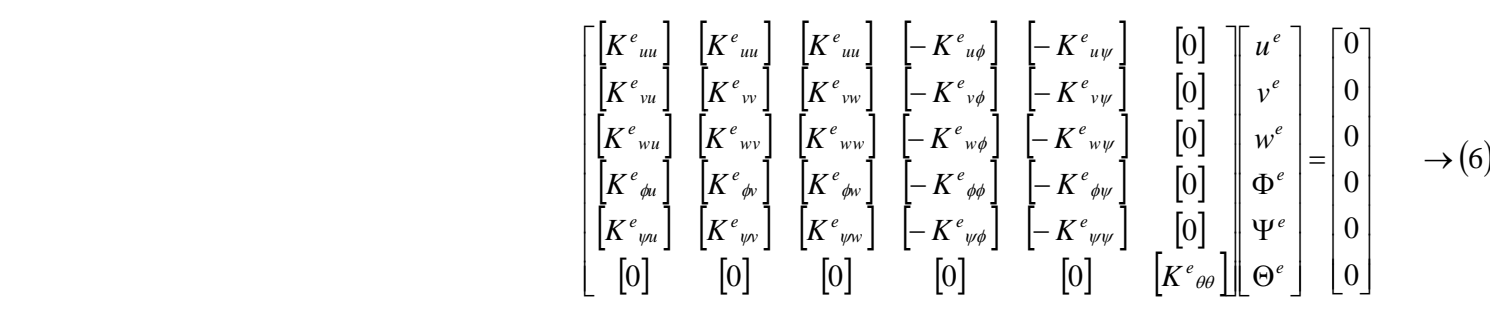

(i.e) $[M]\{\ddot{U}\}+[C]\{\dot{U}\}+[K]\{U\}=0$

$$
\begin{aligned}
& {\left[K^{e_{u u}}\right]=\int_{V}\left[B_{u}\right]^{T}[C]\left[B_{u}\right] d V,} \\
& {\left[K^{e}{ }_{u \theta}\right]=\int_{V}\left[B_{u}\right]^{T}[\beta]\left[N_{\theta}\right]^{T} d V,} \\
& {\left[K^{e_{\phi \theta}}\right]=\int_{V}\left[B_{\phi}\right]^{T}[p]\left[N_{\theta}\right]^{T} d V,} \\
& {\left[K^{e_{u \psi}}\right]=\int_{V}\left[B_{u}\right]^{T}[d]\left[B_{\psi}\right] d V,} \\
& {\left[K^{e_{\psi}}\right]=\int_{V}\left[B_{\psi}\right]^{T}[\tau]\left[N_{\theta}\right]^{T} d V,} \\
& {\left[C^{e}{ }_{\theta u}\right]=\int_{V}\left[N_{\theta}\right]^{T}[\beta]^{T}\left[B_{u}\right] d V,} \\
& {\left[C^{e} \theta \theta=\int_{V}\left[N_{\theta}\right]^{T}[a]\left[N_{\psi}\right] d V,\right.} \\
& \text { Where } d V=2 \pi d r d \theta d z
\end{aligned}
$$

$$
\begin{aligned}
& {\left[K^{e{ }}\right]=\int_{V}\left[B_{u}\right]^{T}[e]\left[B_{\phi}\right] d V} \\
& {\left[\boldsymbol{K}^{e} \phi \phi\right]=\int_{V}\left[\boldsymbol{B}_{\phi}\right]^{T}[\varepsilon]\left[\boldsymbol{B}_{\phi}\right] d \boldsymbol{V}} \\
& {\left[\boldsymbol{K}^{\circ}{ }^{\circ O}\right]=\int_{V}\left[\boldsymbol{B}_{O}\right]^{T}[k]\left[\boldsymbol{B}_{\theta}\right] d \boldsymbol{V}} \\
& {\left[K^{\circ}{ }_{\psi}\right]=\int_{V}\left[\boldsymbol{B}_{\psi}\right]^{T}[\boldsymbol{\mu}]\left[\boldsymbol{B}_{\psi}\right] d \boldsymbol{V}} \\
& {\left[C^{e}{ }_{o \phi}\right]=\int_{V}\left[N_{o}\right]^{T}[p]^{T}\left[B_{\phi}\right] d V} \\
& {\left[C^{e} \theta_{\psi}\right]=\int_{V}\left[N_{\theta}\right]^{T}[p]^{T}\left[B_{W}\right] d V} \\
& {\left[\boldsymbol{M}^{\circ}\right]=\int_{V}[N]^{T}[\rho][N] d V}
\end{aligned}
$$

The equation (6) contains elasticity in Cartesian coordinates and are obtained by using the assumed shape functions solutions of equation (5). The four noded element is considered. Every element in each matrices contained smaller sub-matrices that are multiplied by various type of in-plane functions.
The nondimensional frequencies and the corresponding shape functions are obtained by solving the above Eigen value problem without external forces.

Assemble all element equations, the required frequency equation is,

$$
\begin{aligned}
& -p^{2}[M]\{u\}-i p[C]\{u\}+[K]\{u\}=0 \quad \rightarrow(7) \\
& \text { where }[M]=\left[\begin{array}{ccc}
M_{u u} & 0 & 0 \\
0 & 0 & 0 \\
0 & 0 & 0
\end{array}\right], \quad[C]=\left[\begin{array}{cccc}
0 & 0 & 0 & 0 \\
0 & 0 & 0 & 0 \\
0 & 0 & 0 & 0 \\
C_{\theta u} & -C_{\theta \phi} & -C_{\theta \psi} & C_{\theta \theta}
\end{array}\right] \text { and }[K]=\left[\begin{array}{cccc}
K_{u u} & K_{u \phi} & K_{u \phi} & -K_{u \theta} \\
K_{\phi u} & -K_{\phi \phi} & -K_{\phi \psi} & K_{\phi \theta} \\
K_{\psi u} & -K_{\psi \phi} & -K_{\psi \psi} & K_{\psi \theta} \\
0 & 0 & 0 & K_{\theta \theta}
\end{array}\right] \\
& T_{k j, j}+F_{i}=\rho \ddot{u}_{k} \\
& D_{k, k}=0 \text {, } \\
& \left.\begin{array}{l}
B_{k, k}=0 \\
q_{k, k}+T_{0} \dot{\eta}=0
\end{array}\right] \rightarrow
\end{aligned}
$$
constants values of Cadmium selenide and Cobalt ferrite.

\section{Newmark's finite difference formulation} displacement is vanished. By using these conditions, the equations of motion can be written in the form,
Substitute equation (5) and corresponding shape function values are in (8) and to make integration over the corresponding volume. Equation (6) can be change over to the following Stiffness $[K]$, Damping $[C]$, Mass $[M]$ and force $[F]$ matrices. 


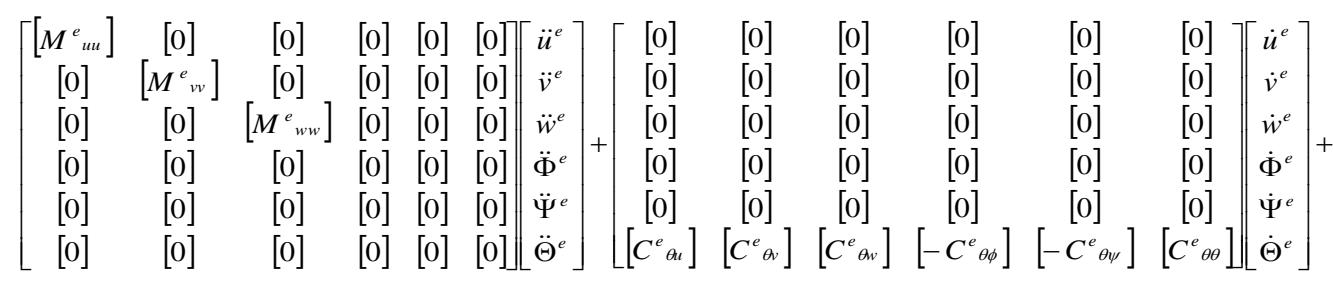

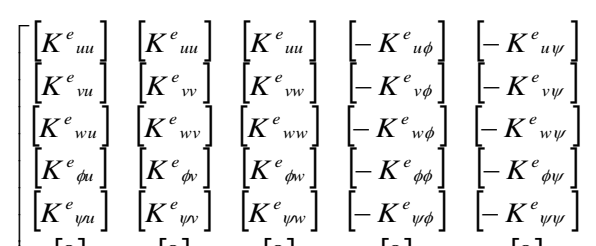

$[0]$

[0]

[0]

[0]

$[0]$ $\left.\begin{array}{c}{[0]} \\ {[0]} \\ {[0]} \\ {[0]} \\ {[0]} \\ {\left[K_{\theta \theta}^{e}\right]}\end{array}\right]\left[\begin{array}{c}u^{e} \\ v^{e} \\ w^{e} \\ \Phi^{e} \\ \Psi^{e} \\ \Theta^{e}\end{array}\right]=\left[\begin{array}{c}F^{e} \\ F^{e} \\ F^{e} \\ F^{e} \\ F^{e} \\ F^{e}\end{array}\right]$ (i.e) $[M]\{\ddot{U}\}+[C]\{\dot{U}\}+[K]\{U\}=[F]$

After getting the global Stiffness, Damping, Mass and force matrices of each element, consider the following shape functions for each element

$$
\begin{aligned}
& \Phi=\left[\begin{array}{ll}
N_{1} & N_{2}
\end{array}\right], U=\left[\begin{array}{c}
u_{i} \\
u_{i+1}
\end{array}\right], \hat{u}=\Phi U \\
& \Phi=\left[\begin{array}{ll}
N_{1} & N_{2}
\end{array}\right], T=\left[\begin{array}{c}
T_{i} \\
T_{i+1}
\end{array}\right], \hat{T}=\Phi T
\end{aligned}
$$

The following dynamic finite element governing equation can be obtained for each element by using linear shape function are

$$
[M]_{e}\{\ddot{\Phi}\}+[C]_{e}\{\dot{\Phi}\}+[K]_{e}\{\Phi\}=[F]_{e}
$$

$$
\text { Where } \quad\{\Phi\}^{T}=\left\{\begin{array}{llll}
\hat{u}_{i} & \hat{T}_{i} & \hat{u}_{i+1} & \hat{T}_{i+1}
\end{array}\right\}^{T}
$$

" $e$ "is $e^{\text {th }}$ element and " $i$ "stand for $i^{\text {th }}$ node. After assembling of all matrices of each of the elements, the coupled thermos electric elastic problem in the form of stochastic frame work can be rewritten as,

$$
\left\{(U)_{t+\Delta t}\right\}=\frac{[K]_{e f f}}{\left([F]_{e f f}\right)_{t}}
$$

$$
\text { Where }[K]_{e f f}=[K]+a_{0} *[M]+a_{1} *[C] \text { and }
$$$$
\left([F]_{e f f}\right)_{t}=[F]+[M] *\left(a_{0} *\left\{(U)_{t}\right\}+a_{2} *\left\{(\dot{U})_{t}\right\}+a_{3} *\left\{(\ddot{U})_{t}\right\}\right)+[C] *\left(a_{1} *\left\{(\dot{U})_{t}\right\}+a_{4} *\left\{(\dot{U})_{t}\right\}+a_{5} *\left\{(\ddot{U})_{t}\right\}\right)
$$

$\left\{(\ddot{U})_{t+\Delta t}\right\}=a_{0} *\left(\left\{(U)_{t+\Delta t}\right\}-\left\{(U)_{t}\right\}\right)-a_{2} *\left\{(\dot{U})_{t}\right\}-a_{3} *\left\{(\ddot{U})_{t}\right\}$

$\left\{(\dot{U})_{t+\Delta t}\right\}=\left\{(\dot{U})_{t}\right\}+a_{6} *\left\{(\ddot{U})_{t}\right\}+a_{7} *\left\{(\ddot{U})_{t+\Delta t}\right\}$

Once the value of displacements $\left\{(U)_{t+\Delta t}\right\}$ at time $t_{t+\Delta t}$ are obtained then solving equation (14) the velocities $\left\{(\dot{U})_{t+\Delta t}\right\}$ and accelerations $\left\{(\ddot{U})_{t+\Delta t}\right\}$ are obtained respectively.

In

equations

(14), (15) and (16), $a_{0}, a_{1}, a_{2}, a_{3}, a_{4}, a_{5}, a_{6}$ and $a_{7}$ are

Newmark parameters and the values are given in Appendix. In Newmark difference method the best convergence can be reached by choosing $\alpha=0.25$ and $\delta=0.5$ in the above parameters.

\section{Static analysis}

In on static vibration boundary conditions, the velocity and acceleration are vanished. By using these conditions, the governing equilibrium equations can be written in the form,

$$
[M]\{\ddot{\Phi}\}+[C]\{\dot{\Phi}\}+[K]\{\Phi\}=[F]
$$

The above equation is in transient nature. By applying the Newmark's integration method, and to approximate the time this method, the functions and its corresponding derivatives are approximated by the following assumptions.

Initially to calculate the value of displacements $\left\{U_{t+\Delta t}\right\}$, the constitute equation can be evaluated at time $t_{t+\Delta t}$ as,

$[M]\left\{(\ddot{U})_{t+\Delta t}\right\}+[C]\left\{(\dot{U})_{t+\Delta t}\right\}+[K]\left\{(U)_{t+\Delta t}\right\}=\left\{(F)_{t+\Delta t}\right\}$

Where $\Delta t=$ time step, $t=$ step number. Rearranging the above equation,

$\left\{(\ddot{U})_{t}\right\}=[M]^{-1} *\left([F]-[C]^{*}\left\{(\dot{U})_{t}\right\}-[K]^{*}\left\{(U)_{t}\right\}\right)$

The displacement matrix $\left\{(U)_{t+\Delta t}\right\}$, velocity matrix $\left\{(\dot{U})_{t+\Delta t}\right\}$ and acceleration matrix $\left\{(\ddot{U})_{t+\Delta t}\right\}$ can be found by using following equations, derivatives and to solve the equations of forced vibration. About
$[K]\{U\}=\{F\}$

\section{Analysis and results}

The values of displacement $\left\{(U)_{t+\Delta t}\right\}$, velocity $\left\{(\dot{U})_{t+\Delta t}\right\}$ and acceleration $\left\{(\ddot{U})_{t+\Delta t}\right\}$ for the materials Cdse/CoFe2o4 are obtained. The terms for non-dimensional are necessary to solve the problem and assumed as follows. The thermo magneto electric elastic terms are assumed by $\operatorname{Ref}[2,3 \& 4]$ and the additional non-dimensional terms were derived based upon the constitute equations. The non-dimensional terms for the infinite solid cylinder analysis are as follows, 


$$
\begin{gathered}
\overline{C_{i j}}=\frac{c_{i j}}{c_{44}}, \quad K=\frac{\varepsilon}{h}, \quad \overline{\beta_{i j}}=\frac{\beta_{i j}}{\beta_{3}}, \overline{p_{i j}}=\frac{p_{i j} c_{44}}{e_{33} \beta_{3}}, \overline{K_{i j}}=\frac{\left(\rho c_{44}\right)^{\frac{1}{2}} K_{i j}}{\beta_{2}^{2} h T_{0}} \\
\overline{\tau_{i j}}=\frac{\tau_{i j} c_{44}}{d_{33} \beta_{3}}
\end{gathered}
$$

The displacement $\left\{(U)_{t+\Delta t}\right\}$, velocity $\left\{(\dot{U})_{t+\Delta t}\right\}$ and acceleration $\left\{(\ddot{U})_{t+\Delta t}\right\}$ are obtained by using MATLAB software. The natural frequencies and static vibrations are obtained by various values of dimensionless wave number. The displacement, velocity and acceleration values are obtained and represented graphically, with different time variants.

\section{Conclusion}

The static, transient and free vibration analysis of an infinite thermo- magneto-electric-elastic solid cylinder is solved by using hybrid numerical method. The cylinder is of $6 \mathrm{~mm}$ class with $\ell=$ $\cos 30^{\circ}$ and $m=\sin 30^{\circ}$. Thickness of the cylinder is taken by $h=0.05 \mathrm{~mm}$. The constitute equations for thermo-magnetoelectric-elastic materials are derived by using Finite element formulations with free/forced vibrations. The coupled mass, stiffness and damping and matrices, the global transient equations are executed in time domains. In static vibration the acceleration and velocity are vanished. The Newmark finite difference method with suitable time step is used and the equilibrium equations are solved.The values of displacement, velocity, acceleration and the natural frequencies are obtained. The numerical illustrations have been carried out for Cadmium selenide and Cobalt ferrite. Solutions are tabulated and illustrate graphically. The nondimensional wave number versus the displacement, velocity and acceleration values are calculated with suitable time variants. Finally, it is observed that in the case of Free vibration, the natural frequencies of thermo magneto-electric-elastic infinite solid cylinder made by the material $\mathrm{CoFe} 2 \mathrm{o} 4$ is significantly greater than that of Cdse material. The damping effect analyzed through the imaginary part of complex frequencies. In the case of Transient, different values of nondimensionl wave number, if the time domain increased when the values of the displacement, velocity and acceleration of $\mathrm{CoFe} 2 \mathrm{o} 4$ material is significantly greater than that of Cdse material. In the case of static,different values of nondimensionl wave number, if the time domain increased when the values of the displacement of $\mathrm{CoFe} 2 \mathrm{o} 4$ material is significantly greater than that of Cdse material.

\section{Numerical illustration and figures}

\section{In Static State}

\begin{tabular}{|r|c|c|}
\hline \multicolumn{1}{|c|}{$\boldsymbol{\epsilon}$} & \multicolumn{2}{|c|}{ Displacement } \\
\cline { 2 - 3 } & Cdse & CoFe204 \\
\hline 0.2 & $0.181976-0.12228 \mathrm{i}$ & $0.35957-0.14607 \mathrm{i}$ \\
\hline 0.4 & $0.635618-0.16709 \mathrm{i}$ & $0.65289-0.52207 \mathrm{i}$ \\
\hline 0.45 & $0.725172+0.17587 \mathrm{i}$ & $0.86797-0.15455 \mathrm{i}$ \\
\hline 0.6 & $0.88758-0.186163 \mathrm{i}$ & $0.97829-0.94006 \mathrm{i}$ \\
\hline 0.7 & $0.93236-0.215972 \mathrm{i}$ & $1.34113+1.37515 \mathrm{i}$ \\
\hline 0.8 & $1.52199+0.616027 \mathrm{i}$ & $1.84732-1.20416 \mathrm{i}$ \\
\hline 1 & $2.12598-0.715699 \mathrm{i}$ & $2.70513+0.74629 \mathrm{i}$ \\
\hline 2 & $2.65865+0.891626 \mathrm{i}$ & $3.25022-0.33856 \mathrm{i}$ \\
\hline 2.7 & $3.39057+1.163713 \mathrm{i}$ & $3.86912-0.16338 \mathrm{i}$ \\
\hline 4 & $3.783265-1.198454 \mathrm{i}$ & $4.33984+0.42162 \mathrm{i}$ \\
\hline
\end{tabular}

\begin{tabular}{|c|c|c|c|c|}
\hline \multirow{3}{*}{$\boldsymbol{\epsilon}$} & \multicolumn{4}{|c|}{ Displacement } \\
\hline & \multicolumn{2}{|c|}{$T=0.1$} & \multicolumn{2}{|c|}{$\mathrm{T}=0.2$} \\
\hline & Cdse & CoFe204 & Cdse & CoFe204 \\
\hline 0.2 & $0.17385-0.14506 \mathrm{i}$ & $0.20752+0.58209 \mathrm{i}$ & $0.25859+0.29842 \mathrm{i}$ & $0.28412-0.29253 \mathrm{i}$ \\
\hline 0.4 & $0.25789-0.11045 \mathrm{i}$ & $0.28679-0.69887 i$ & $0.33439+0.26742 \mathrm{i}$ & $0.37911+0.23485 \mathrm{i}$ \\
\hline 0.45 & $0.36597-0.37645 \mathrm{i}$ & $0.39195+0.68642 \mathrm{i}$ & $0.40287-0.37169 \mathrm{i}$ & $0.44421-0.42332 \mathrm{i}$ \\
\hline 0.6 & $0.43747+0.49966 \mathrm{i}$ & $0.49815+0.68991 \mathrm{i}$ & $0.48592+0.49439 \mathrm{i}$ & $0.52654+0.40285 \mathrm{i}$ \\
\hline 0.7 & $0.47353+0.54084 \mathrm{i}$ & $0.56147+0.70353 \mathrm{i}$ & $0.52616-0.50687 \mathrm{i}$ & $0.62674+0.45896 \mathrm{i}$ \\
\hline 0.8 & $0.56538-0.52136 \mathrm{i}$ & $0.61119-0.80825 \mathrm{i}$ & $0.67404+0.43702 \mathrm{i}$ & $0.70067+0.48664 \mathrm{i}$ \\
\hline 1 & $0.66715+0.41488 \mathrm{i}$ & $0.76455+0.88199 \mathrm{i}$ & $0.74361-0.44021 \mathrm{i}$ & $0.84259+0.39632 \mathrm{i}$ \\
\hline 2 & $0.69415+0.49345 \mathrm{i}$ & $0.83675-0.88844 \mathrm{i}$ & $0.83889-0.35139 \mathrm{i}$ & $0.87208+0.43583 \mathrm{i}$ \\
\hline 2.7 & $0.78849+0.40812 \mathrm{i}$ & $0.86049+0.98158 \mathrm{i}$ & $0.86654+0.35494 \mathrm{i}$ & $0.90776-0.41547 \mathrm{i}$ \\
\hline & & & & \\
\hline
\end{tabular}

In Transient State

\begin{tabular}{|c|c|c|c|c|}
\hline \multirow{3}{*}{$\boldsymbol{E}$} & \multicolumn{4}{|c|}{ ocity } \\
\hline & \multicolumn{2}{|c|}{$T=0.1$} & \multicolumn{2}{|c|}{$T=0.2$} \\
\hline & Cdse & $\mathrm{CoFe204}$ & Cdse & CoFe204 \\
\hline 0.2 & $0.31656+0.13335 i$ & $0.36402-0.23719 \mathrm{i}$ & $0.36741-0.27547 \mathrm{i}$ & $0.48047+0.33726 \mathrm{i}$ \\
\hline 0.4 & $0.52734+0.12334 \mathrm{i}$ & $0.57281-0.23719 \mathrm{i}$ & $0.54796-0.93767 \mathrm{i}$ & $0.61385+0.22356 \mathrm{i}$ \\
\hline 0.45 & $0.56981-0.46883 \mathrm{i}$ & $0.65236+0.18112 \mathrm{i}$ & $0.61424+0.26668 \mathrm{i}$ & $0.71812+0.43727 \mathrm{i}$ \\
\hline 0.6 & $0.63598-0.468832 \mathrm{i}$ & $0.72882+0.67454 \mathrm{i}$ & $0.71948-0.93766 \mathrm{i}$ & $0.78442+0.43726 \mathrm{i}$ \\
\hline 0.7 & $0.71108+0.43336 \mathrm{i}$ & $0.83364+0.67457 \mathrm{i}$ & $0.83941-0.93768 \mathrm{i}$ & $0.86372-0.47859 \mathrm{i}$ \\
\hline 0.8 & $0.77965-0.368831 \mathrm{i}$ & $0.88438+0.67453 \mathrm{i}$ & $0.92532+0.26667 \mathrm{i}$ & $0.98192+0.33727 \mathrm{i}$ \\
\hline 1 & $0.85828+0.23335 \mathrm{i}$ & $0.94805+0.67454 \mathrm{i}$ & $0.95512+0.71597 \mathrm{i}$ & $1.39095+0.64763 \mathrm{i}$ \\
\hline 2 & $0.91656+0.13336 i$ & $0.96096+0.77456 \mathrm{i}$ & $1.23983-0.93767 \mathrm{i}$ & $1.82008-0.11859 \mathrm{i}$ \\
\hline 2.7 & $0.94274+0.13336 \mathrm{i}$ & $1.2973+0.67459 \mathrm{i}$ & $1.8547+0.26668 \mathrm{i}$ & $2.45712-0.11856 \mathrm{i}$ \\
\hline 4 & $0.98332+0.13335 \mathrm{i}$ & $1.9219+0.67454 \mathrm{i}$ & $2.6623+0.26688 \mathrm{i}$ & $2.89935+0.90558 \mathrm{i}$ \\
\hline
\end{tabular}

\begin{tabular}{|c|c|c|c|c|}
\hline \multirow[b]{3}{*}{$\boldsymbol{\epsilon}$} & \multicolumn{4}{|c|}{ Acceleration } \\
\hline & \multicolumn{2}{|c|}{$T=0.1$} & \multicolumn{2}{|c|}{$\mathrm{T}=0.2$} \\
\hline & Cdse & $\mathrm{CoFe} 204$ & Cdse & CoFe204 \\
\hline 0.2 & $0.23982-0.53765 \mathrm{i}$ & $0.26841+0.60589 \mathrm{i}$ & $0.36741-0.47557 \mathrm{i}$ & $0.39601+0.60463 \mathrm{i}$ \\
\hline 0.4 & $0.47966-0.43766 \mathrm{i}$ & $0.56402-0.41859 \mathrm{i}$ & $0.52663+0.46667 \mathrm{i}$ & $0.58385+0.52357 \mathrm{i}$ \\
\hline 0.45 & $0.53962-0.43766 \mathrm{i}$ & $0.60952-0.41859 \mathrm{i}$ & $0.61425+0.46678 \mathrm{i}$ & $0.66377+0.47235 \mathrm{i}$ \\
\hline 0.6 & $0.61899+0.36667 \mathrm{i}$ & $0.64414+0.43726 \mathrm{i}$ & $0.68994+0.26678 \mathrm{i}$ & $0.72355+0.40936 \mathrm{i}$ \\
\hline 0.7 & $0.68582+0.51597 \mathrm{i}$ & $0.73874+0.50559 \mathrm{i}$ & $0.72216+0.46866 \mathrm{i}$ & $0.77907+0.48725 \mathrm{i}$ \\
\hline 0.8 & $0.72533+0.56667 \mathrm{i}$ & $0.79872+0.50559 \mathrm{i}$ & $0.75325+0.56667 \mathrm{i}$ & $0.81922+0.53727 \mathrm{i}$ \\
\hline 1 & $0.83166+0.46667 \mathrm{i}$ & $0.89114+0.42049 \mathrm{i}$ & $0.86125+0.46320 \mathrm{i}$ & $0.91004-0.51859 \mathrm{i}$ \\
\hline 2 & $0.91024+0.71599 \mathrm{i}$ & $0.94805+0.83727 \mathrm{i}$ & $0.96334+0.56651 \mathrm{i}$ & $0.98201-0.97889 \mathrm{i}$ \\
\hline 2.7 & $0.98547+0.58667 \mathrm{i}$ & $1.188-0.54972 \mathrm{i}$ & $1.4521-0.25841 \mathrm{i}$ & $1.95705+0.90558 \mathrm{i}$ \\
\hline 4 & & & & \\
\hline
\end{tabular}




\section{In Free vibration}

\begin{tabular}{|r|c|c|}
\hline \multicolumn{1}{|c|}{$\boldsymbol{\epsilon}$} & \multicolumn{2}{|c|}{ Frequency } \\
\cline { 2 - 3 } & Cdse & CoFe2o4 \\
\hline 0.2 & $0.30322457-0.1097239 \mathrm{i}$ & $0.416517+0.124592 \mathrm{i}$ \\
\hline 0.4 & $0.43469401-0.1775241 \mathrm{i}$ & $0.501383+0.4905423 \mathrm{i}$ \\
\hline 0.45 & $0.46385599-0.1886535 \mathrm{i}$ & $0.624275-0.518148 \mathrm{i}$ \\
\hline 0.6 & $0.53948156-0.2322687 \mathrm{i}$ & $0.810398-0.210655 \mathrm{i}$ \\
\hline 0.7 & $0.55015719-0.317568 \mathrm{i}$ & $0.867063-0.586072 \mathrm{i}$ \\
\hline 0.8 & $0.5977587+0.107401 \mathrm{i}$ & $0.872373-0.612630 \mathrm{i}$ \\
\hline 1 & $0.6592016-0.23674 \mathrm{i}$ & $0.910399-0.510655 \mathrm{i}$ \\
\hline 2 & $1.08611619-0.10179798 \mathrm{i}$ & $1.442395+0.543578 \mathrm{i}$ \\
\hline 2.7 & $1.1015069-0.87832 \mathrm{i}$ & $2.0541178-0.270685 \mathrm{i}$ \\
\hline 4 & $1.31437205+0.743434$ & $2.8305593+0.884324 \mathrm{i}$ \\
\hline
\end{tabular}

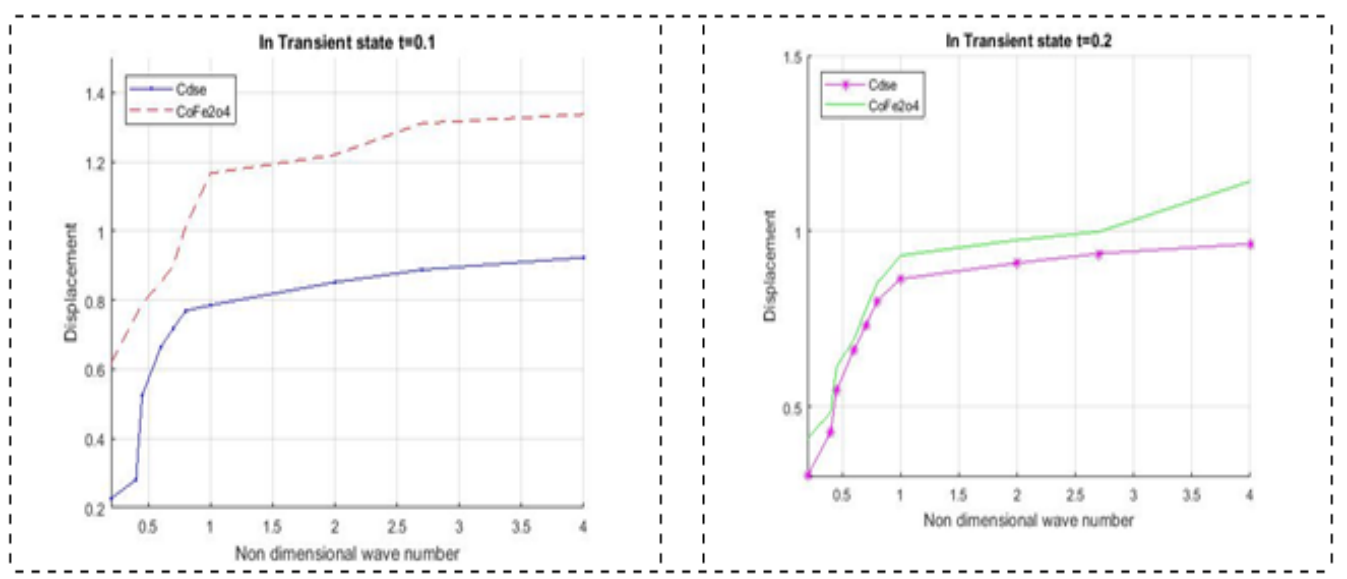

Figure 1: Non dimensionl wave number Vs Displacement with transient state

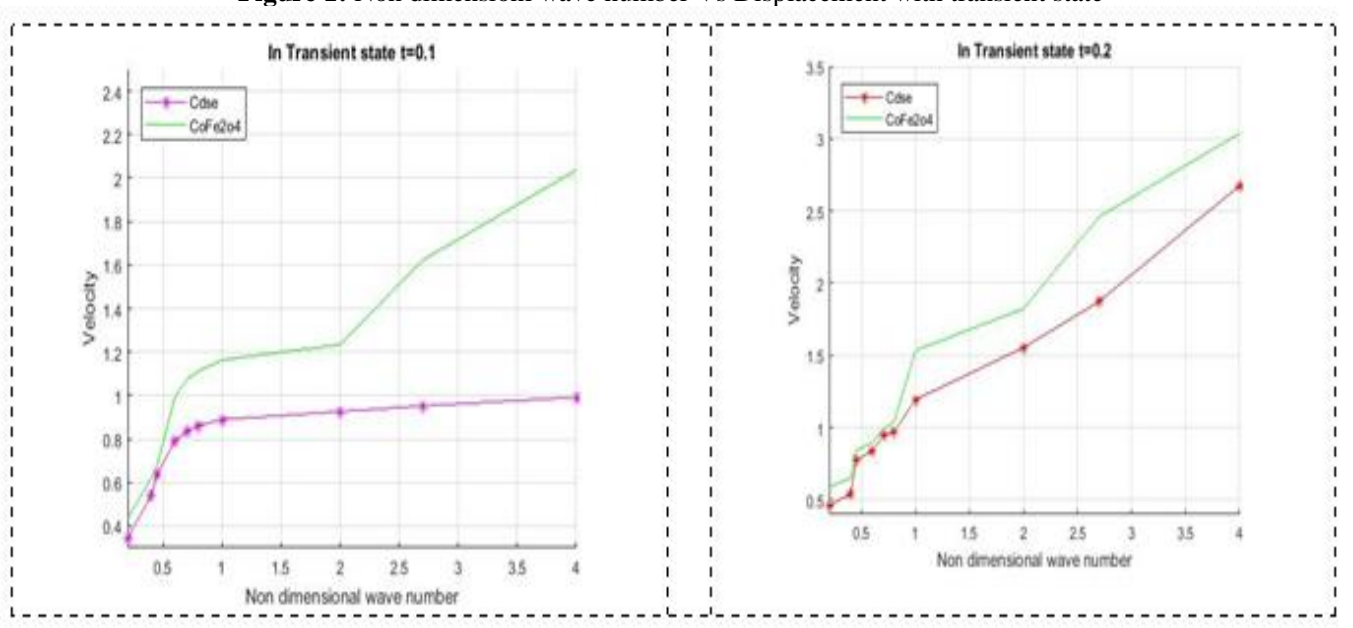

Figure 2: Non dimensional wave number Vs Velocity with transient state

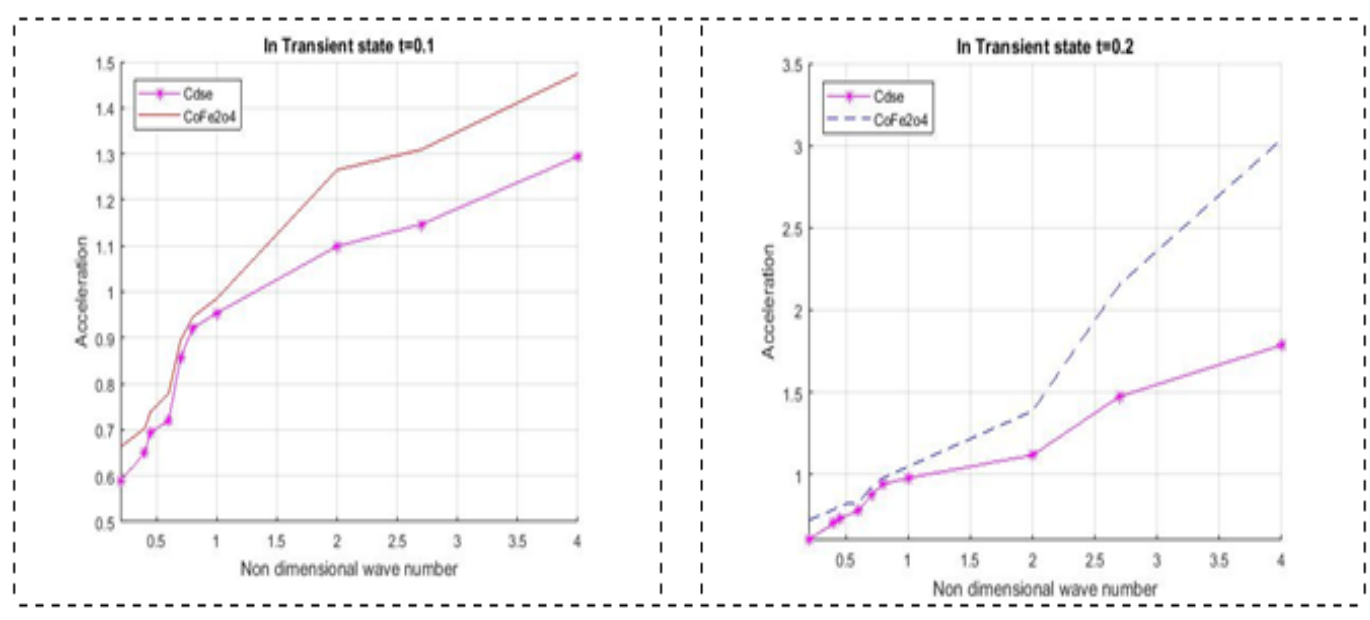

Figure 3: Non dimensional wave number Vs Acceleration with transient state 


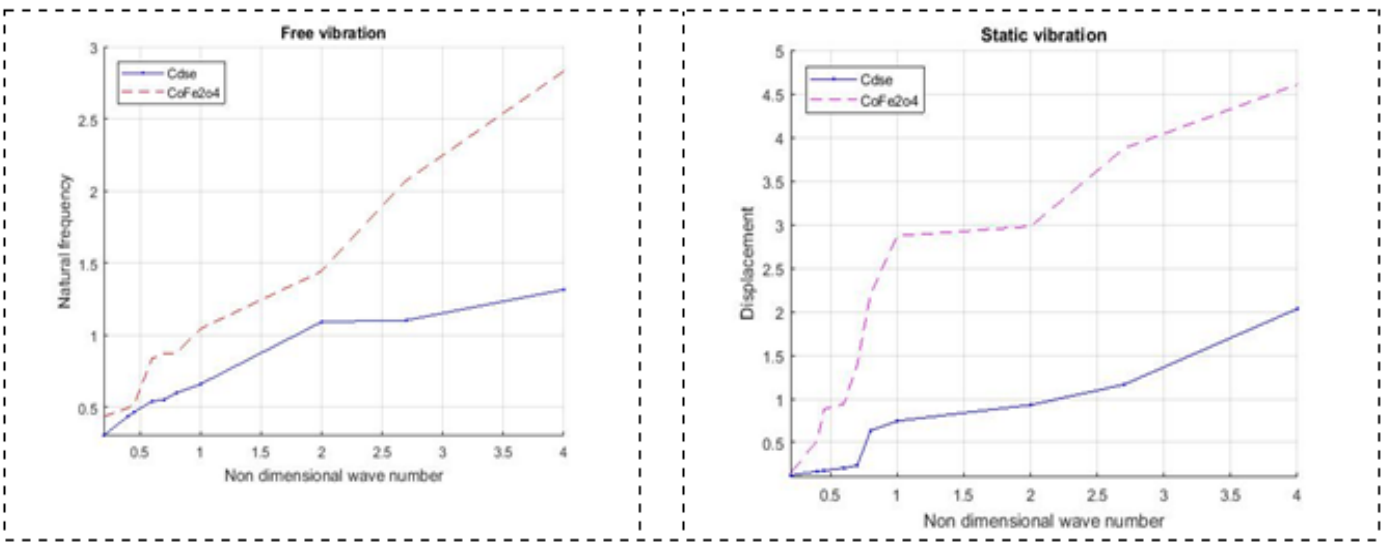

Figure 4: Non dimensional wave number Vs natural frequency with free vibration Figure 5: Non dimensional wave number Vs displacement with static Vibration

\section{References}

[1] [1] Mindlin RD, "High frequency vibration of Piezoelectric crystal plates", Int. J. solid Structures, Vol.8, (1972), pp.895-906.

[2] [2] Paul HS \& Nelson VK, "Wave propagation in Piezo composite plate", Proc. Indian. Sci. Acadamy, Vol.61, No.3\&4, (1995), pp. $22-228$

[3] [3] Nelson VK \& Karthikeyan S, "Vibration of pyro electric sandwich plate", i-managers Journal on Future Engineering Technology, Vol.6, No.1, (2010).

[4] Paul HS \& Ranganathan K, "Free vibrations of pyro electric laye hexagonal (6mm) class", JASA, Vol.78, No.2, (2011), pp.895-397.

[5] Paul HS \& Raman GV, "Vibration of pyro electric plates", JASA, Vol.90, No.4, (1991), pp.1729-1732.

[6] Paul HS, Raju DP \& Balakrishnan TR, "Free vibrations of Piezo electric layer of Hexagonal $(6 \mathrm{~mm})$ class", Int. J. Engg. Sci. Vol.21.No.6, (1983), pp.69-704.

[7] Ganesan N, Kumaravel A \& Rajusethuraman, "Finite Elemen Modelling of a Layered, Multiphase Magneto electro elastic cylinder subjected to an ax symmetric temperature Distribution", Journal of mechanics of material and structures, Vol.2, No.4, (2007).

[8] George RB, "Galerkin finite element derivation for vibration of a thermopiezo electric structure", Journal of sound and vibration, Vol.294, (2006), pp.362-367

[9] Seyed MH \&Farzad S, "Transient analysis of thermo elastic waves in thick hollow cylinders using a stochastic hybrid numerical method, considering Gaussian mechanical properties", Journal of Applied Mathematical modelling, Vol.35, (2011), pp.4697-4714.

[10] Chen WQ., Wang HM., Bao RH, "On calculating dispersion curves of waves in a functionally graded elastic plate", Composite structures, Vol.81, (2007), pp.233-242.

[11] Seyed MH, Jan S and Vladimir S, "Meshless local petrov-Galerkin method for coupled thermo elasticity analysis of a functionally graded thick hollow cylinder", Engineering analysis with Boundary Elements, Vol.35, (2011), pp.827-835.

[12] Hosseini SM, Sladek J and Sladek V, "Thermoelastic wave propagation in functionally graded materials using meshless local petrov-Galerkin (MLPG) method", $16^{\text {th }}$ International conference on composite structures, (2011).

[13] Pan E and Heyliger PR, "Exact solutions for magneto electro elastic laminates in cylindrical bending", International journal of solids and structures, Vol.40, (2003), pp.6859-6876.

[14] Pan E, "Exact solution for simply supported and multilayered Magneto-Electro-Elastic plates", ASME, Vol.68, (2001), pp.608618

[15] Reddy JN, "Energy and variational methods in applied mechanics", Wiley, New York, (1984).

[16] Jochen A, Carstencarstensen \& Stefan A.F, "Remarks around 50 lines of MATLAB: short finite element implementation, Numerical algorithms, Vol.20, (1999), pp.117-137.

\section{Appendix}

The strain displacement, electric potential $\phi$ and heat flux

$q_{i, j}$

$$
\begin{gathered}
S_{1}=\frac{\partial u}{\partial r}, S_{2}=\frac{1}{r}\left(\frac{\partial v}{\partial \theta}+u\right), S_{3}=\frac{\partial w}{\partial z}, \\
S_{4}=\frac{\partial v}{\partial z}+\frac{1}{r}\left(\frac{\partial w}{\partial \theta}\right), S_{1}=\frac{\partial w}{\partial r}+\frac{\partial u}{\partial z}, \\
S_{4}=\frac{\partial v}{\partial r}+\frac{1}{r}\left(\frac{\partial u}{\partial \theta}\right)-\frac{v}{r} \\
\mathrm{E}_{1}=-\frac{\partial \phi}{\partial r}, \mathrm{E}_{2}=-\frac{1}{r} \frac{\partial \phi}{\partial \theta}, \mathrm{E}_{1}=-\frac{\partial \phi}{\partial z} \quad \text { and } \\
q_{1}=-K_{11} \frac{\partial T}{\partial r}, q_{2}=-K_{22} \frac{\partial T}{\partial \theta}, q_{3}=-K_{33} \frac{\partial T}{\partial z}
\end{gathered}
$$

The material constants for crystal class $6 \mathrm{~mm}$ and New mark Parameters:

$$
\begin{aligned}
& c_{i j}=\left[\begin{array}{cccccc}
c_{11} & c_{12} & c_{13} & 0 & 0 & 0 \\
c_{12} & c_{11} & c_{23} & 0 & 0 & 0 \\
c_{13} & c_{23} & c_{33} & 0 & 0 & 0 \\
0 & 0 & 0 & c_{44} & 0 & 0 \\
0 & 0 & 0 & 0 & c_{44} & 0 \\
0 & 0 & 0 & 0 & 0 & c_{66}
\end{array}\right], \text { where } c_{66}=\left(\frac{c_{11}-c_{12}}{2}\right) \beta_{i j}=\left[\begin{array}{c}
\beta_{1} \\
\beta_{2} \\
\beta_{3} \\
0 \\
0 \\
0
\end{array}\right], p_{j}=\left[\begin{array}{c}
0 \\
0 \\
p_{3}
\end{array}\right] \\
& e_{k j}=\left[\begin{array}{cccccc}
0 & 0 & 0 & 0 & e_{15} & 0 \\
0 & 0 & 0 & e_{15} & 0 & 0 \\
e_{31} & e_{31} & e_{33} & 0 & 0 & 0
\end{array}\right], \quad \varepsilon_{k l}=\left[\begin{array}{ccc}
\varepsilon_{11} & 0 & 0 \\
0 & \varepsilon_{11} & 0 \\
0 & 0 & \varepsilon_{33}
\end{array}\right] \\
& a_{0}=\frac{\left(\alpha *(\Delta t)^{2}\right),}{(\alpha)} a_{1}=\frac{\delta}{(\alpha * \Delta t)} \\
& a_{2}=\frac{1}{(\alpha * \Delta t)}, a_{3}=\frac{1}{(2 * \alpha)}-1 \\
& \left.a_{4}=\frac{\delta}{\alpha}-1, \quad \frac{\Delta t}{2}\right) *\left(\frac{\delta}{\alpha-2}\right) \\
& a_{6}=\frac{\Delta t *(1-\delta),}{a_{7}}=\delta * \Delta t
\end{aligned}
$$

